\title{
Simultaneous COVID -19 and Cytomegalovirus infection in Renal Allograft Recipient: Case Report
}

Tania Mahbub*1, Elena Haque 2 , Salina Akter ${ }^{3}$

DOI: https://doi.org/10.3329/bccj.v9i1.53061

\begin{abstract}
:
Coronavirus Disease 2019 (COVID-19) has become a pandemic since March 2020.Kidney-transplant recipients appear to be at particularly high risk for critical COVID-19 illness due to chronic immunosuppression and coexisting conditions. We report a case of a young lady who being a renal allograft recipient developed concomitant COVID-19 and Cytomegalovirus (CMV) infection.
\end{abstract}

\section{Introduction:}

The 2019 novel coronavirus disease (COVID-19) was first detected in Wuhan, Hubei Province, China, in late 2019. Since then, COVID-19 has spread to more than 200 countries in the world, and a global pandemic has been declared by the World Health Organization(WHO $)^{1}$.Disease epidemiology and clinical presentation has been evolving since the outbreak of the novel disease.As immunocompromised hosts Renal Allograft recipients (RARs) are more susceptible for COVID-19infection due to presence of multiple comorbidities, exposure to long-term immunosuppression, and residual Chronic Kidney Disease (CKD). However, till to date little is known about the risk factors, clinical presentation, diagnostic criteria, therapeutic protocols, and outcome of RAR who become infected by SARS-CoV-2. ${ }^{10}$

According to a study on transplant recipients with COVID-19 infection the most common presenting symptom was fever (77\%) followed by cough (58\%). This study found $91 \%$ patient needed hospitalization and only $9 \%$ were stable and received treatment on outpatient's basis ${ }^{2}$.

AKI has been described with COVID-19 infections in up to $15 \%$ patients and occurrence of proteinuria or hematuria has been reported also. In the case series described by Banerjee et. al. $^{3}$ they observed that 4 out of 7 patients had AKI $(57 \%)^{3}$.Another study in transplant patients found higher risk of AKI with COVID-19 infection, compared with $29 \%$ AKI in critically ill patients of general population in Wuhan, China. ${ }^{4,5}$

Elias M et.al. Shown 42\% patients with COVID- 19 developed $\mathrm{AKI}^{2}$.

1. Consultant, Nephrology, United Hospital Limited, Dhaka, Bangladesh

2. Specialist, Nephrology, United Hospital Limited, Dhaka, Bangladesh

3. Junior Consultant, Nephrology,United Hospital Limited, Dhaka, Bangladesh

*Corresponding Author:

Dr. Tania Mahbub

MD (Nephrology)

Consultant, Dept. of Nephrology,

United Hospital Ltd, Gulshan 2,Dhaka.

Email: taniaimran1@yahoo.com
Kidney disease improving global outcomes (KDIGO) stated mortality rate related to COVID-19 disease in kidney transplant population was of $1 \%$. Sixteen of the $66(24 \%)$ patients who were COVID-19 positive died during the pandemic period. Mortality was higher in the invasive mechanical ventilation group: 11 of $15(73 \%)$ in the invasive mechanical ventilation group and five of $51(10 \%)$ in the non-invasive ventilation group. At the end of follow-up, 50 $(76 \%)$ patients had recovered. ${ }^{6}$

With regard to prognostic blood tests including lymphocyte counts and serum levels of D dimer, ferritin and troponin are important prognostic factors. Banerjee et.al. noted very high ferritin and D dimer levels in their patients. They suggest that $\mathrm{D}$ dimer, ferritin, and troponin should be measured in all patients with severe COVID-19 infection on admission and subsequently in those who are not showing clinical improvement. ${ }^{3}$

The indication for antiviral therapy for RAR is also uncertain and there is concern about drug to drug interaction and there are no approved drugs in this regard to date ${ }^{7}$.

The treatment of RAR with COVID-19 infection is unclear. Banerjee et.al ${ }^{3}$. at their report they could not make firm recommendation due to small size cases however they suggest that anti proliferative agents (myco phenolate mofetil and azathioprine) should be stopped at the time of admission to hospital, dose of prednisolone should be either unchanged or increased, and tacrolimus dose should be reduced. They also suggest in severe infections (requiring intubation and ventilation), an argument can be made for stopping calcineurin inhibitors completely while maintaining corticosteroid therapy. There is concern as there is risk of rejection with reduction in immunosuppression but given the high mortality rate of COVID-19 infection in hospitalized patients, they put emphasis on keeping their patients alive with a careful case-by-case assessment of risks versus benefits of continuing immunosuppression.

Due to limited number of cases and lack of large-scale clinical trial optimal COVID-19 management in RAR patient is still under debate, and the therapeutic approach still lacks significant evidence. Apart from symptomatic support therapy, neither specific treatment nor best practice guidelines still exist for the management for RARs with COVID-19. 
However, enhancement of personal protective precautions, early identification, and timely management of affected patients seems to be crucial, particularly in this special subgroup.

Immuno compromised patients often exhibit atypical presentations of viral diseases. A strong clinical suspicion is crucial in such high-risk group of patients. Immunocompromised individuals may exhibit atypical features of viral infection therefore high index of suspicion is required for such high-risk group of patients. SARS-CoV-2 may cause a variety of symptoms including upper respiratory (sore throat), lower respiratory (cough and dyspnea), constitutional (fever, malaise, myalgia), gastrointestinal (nausea, vomiting, abdominal pain, diarrhea), or a combination of them. Sometimes immune compromised hosts may have two or more infection at a time. When complicated with another infection management becomes more challenging. ${ }^{8}$

Herein we report a case of COVID-19 infection in a RAR who presented with acute gastrointestinal symptom along with electrolyte abnormalities and later found to have concomitant COVID-19 and CMV infection. To our knowledge this is the first case having simultaneous COVID-19 and CMV infection.

\section{Case presentation:}

A 27 years old lady with background history of Type 1 DM, Renal Allograft Recipient from her mother on 2016 having history of Chronic Allograft Nephropathy(CAN) in 2018 presented at out patient department with severe acute gastrointestinal symptoms in the form of nausea and vomiting with 3 days $\mathrm{H} / \mathrm{O}$ generalized weakness, mild fever and cough.Her nausea and vomiting were so intense that she was unable to take sip of water. On examination she appeared extremely unwell. BP was $80 / 50 \mathrm{~mm} \mathrm{Hg}$, pulse 57 per min. SPO2 $97 \%$ in room air. Urgent investigation yielded hypoglycemia (blood glucose $2.8 \mathrm{mmol} / \mathrm{L}$ ) and severe hypokalemia (K $1.9 \mathrm{mmol} / \mathrm{l}$ ) with normal anion gap metabolic acidosis. Initial chest X ray (CXR) was normal. Having a high index of suspicion, we sent nasal swab for COVID RT-PCR and blood for Quantitative CMV DNA. After correction of dehydration, hypotension and hypoglycemia on the same day evening she was found to be COVID 19 positive. She was on regular Tacrolimus, Mycofenolate Mofetil (MMF), Evarilumus, Prednisone daily. Due to her intense nausea and vomiting she was kept nothing per oral and MMF and Evarilumus were kept on hold. She was started on injectable steroid along with proton pump inhibitor (PPI), inj. Meropenum intravenous followed by oral potassium, subcutaneous enoxaparin. Tacrolimus was continued according to trough level. Her GI symptoms resolved within 2 days and she started taking food orally thereafter. Although her renal function was deteriorating from baseline. She had satisfactory urine output. Intravenous steroid was switched to oral Prednisolone and gradual institution of MMF was planned. We didn't treat her with antiviral as she responded to initial therapy.However, after 6 days of admission her CMV quantitative DNA came positive.
After an initial clinical and biochemical improvement, she again developed high grade fever 105 degree Fahrenheit with rigor alongside intractable coughing and $\mathrm{SpO} 2$ went down to 94\%.Her D-dimer, Ferritin and CRP went up from initial value and lymphocyte was further decreased with pancytopenia and CXR showed bilateral patchy opacity. HRCT also revealed B/L ground glass opacity suggestive of COVID pneumonia. Assuming Cytokine Releasing Syndrome (CRS) we stared inj. Dexamethasone, Tab. Favipiravir and she was transfused with 2 units of convalescent plasma and we put her on oral Valgancyclovir according to eGFR. Eventually she became afebrile and other symptoms were also improved after a day along with improvement of all biochemical parameters.Throughout this period, she maintained satisfactory urine output and s. creatinine also fell down to baseline. She was discharged after 2 weeks with an advice for home isolation till she became COVID-19 RT PCR negative.

She was found to be negative for COVID-19 after 3 weeks of initial presentation. CMV-DNA became undetected after 4 weeks of starting treatment.

Now she is on regular follow-up in renal OPD and maintaining stable baseline renal function and immunosuppressive medications have been readjusted.

\section{Discussion:}

Our patient predominantly had gastrointestinal symptoms along with fever and mild cough.

Banerjee et.al. reported seven cases of COVID-19 in kidney transplant recipients (median age 54), three females, from a cohort of 2082 managed transplant follow-up patients over a six-week period in three south London hospitals. Two of seven patients presented within three months of transplantation. Overall, two were managed on an out-patient basis, but the remaining five required hospital admission, four in intensive care units. All patients displayed respiratory symptoms and fever. Other common clinical features included hypoxia, chest crepitation, lymphopenia and high C-reactive protein. Thus, their findings suggest COVID-19 infection in kidney transplant patients may be severe, requiring intensive care admission. The symptoms were predominantly respiratory and associated with fever. In another study by Akalin et.al. Shows that the most common initial symptom was fever in $58 \%$, and diarrhea was observed in $22 \%$. Eight patients who were in stable condition without major respiratory symptoms $22 \%$ were monitored at home, $78 \%$ were admitted to the hospital. $96 \%$ had radiographic findings that were consistent with viral pneumonia, and 39\% received mechanical ventilation. ${ }^{8}$

Banerjee et. al. reported three of seven patients had chronic kidney disease stage 4 to 5 , with 1 recovering at home and 1 requiring hospital admission but recovering without needing ITU admission ${ }^{3}$. The remaining 4 patients had chronic kidney disease stage 3 , of which 2 had severe disease requiring intubation and ventilation and 1 of them died. Both patients who had severe COVID-19 including the one who died had diabetes mellitus. Our patient belonged to high risk group as she had Type 1 diabetes and Chronic Allograft Nephropathy. 
Later developed AKI on her compromised graft function along with severe hypokalemia and metabolic acidosis which further complicated her situation.

Banerjeeet. al. observed a very high D Dimer, ferritin, cardiac troponin and lymphopenia in their patients ${ }^{3}$. Our patient had also high $\mathrm{D}$ dimer, Ferritin and Lymphocytopenia at presentation later. However, her troponin I was normal.

KDIGO suggests the primary change in immunosuppression regimen in the majority of patients was the complete cessation of antimetabolites (MMF, mycophenolic acid, or azathioprine especially in the invasive mechanical group (antimetabolites were stopped in all patients whose initial treatment included antimetabolites),while continuing tacrolimus (with a goal trough of 4-6 ng/ml) or cyclosporin (with a goal trough of $400-600 \mathrm{ng} / \mathrm{ml}$ ) and the baseline prednisone in those individuals who were on maintenance prednisone. ${ }^{6}$

According to Banerjeee et. al. managing immunosuppression in transplant patients with COVID-19 infection is challenging and should take into account age, severity of COVID-19 infection, associated comorbidities, and time post-transplant. In transplant patients with mild to moderate infections, the usual practice is to continue or make reductions in the dose of immunosuppressive drugs, but this approach might lead to high mortality in patients admitted to hospital with COVID-19 infection. In their study Immunosuppression was modified in six out of seven patients. ${ }^{3}$

In our patient we modified the immunosuppression that is temporarily hold MMF and evarulimus and intensify the dose of steroid. Due to intense vomiting the trough level of tacrolimus went down to $1 \mathrm{pg} / \mathrm{l}$ therefore we gradually increases the dose to achieve target level of 4 to $6 \mathrm{pg} / \mathrm{l}$.

In addition, COVID-19 patients tend to be hypercoagulable, and prophylactic therapy with low molecular weight heparin or low-dose aspirin is strongly recommended. Apixaban has also be used for this purpose when D-dimer levels were higher than $3.0 \mathrm{microg} / \mathrm{mL} .{ }^{3} \mathrm{We}$ put our patient on $\mathrm{S} / \mathrm{C}$ Enoxaparin from day 1 as D-dimer was high at presentation. We did not started antiviral in our patient as she responded to the initial treatment in the form of modification of immunosuppressive and correction of hypovolemia and electrolyte imbalance. But when she again developed fever with features of CSS, we put her on Favipiravir, dexamethasone and transfused 2 units of convalescent plasma.

\section{Conclusion:}

Our patient not only suffered from COVID-19 related illness, more so, she suffered a concomitant CMV infection which further complicated her disease process and put her life in danger and made the treatment more challenging. After her initial improvement when she again developed high grade fever then the treatment was more challenging as both COVID-19 and CMV were positive and her condition was deteriorating. COVID can produce fever and cytokine release syndrome which may produce similar symptom also CMV infection can produce such symptoms. But the therapeutic challenge was CMV infection needs a reduction of immunosuppressive agent and CRS needs an augmentation of immunosuppressive agent, especially steroid. As cytokine cannot be measured in our center therefore it was not possible at that time to determine which infection responsible for deterioration of her condition after an initial improvement. Another challenge we met as there was no established recommendation for treatment of COVID-19 infection in RAR,more so, a compromised renal function further complicated the situation to treat her with antiviral. However, considering her life risk we targated to treat both the infection simultaneously. For COVID she got favipiravir, inj dexamethasone, convalescence plasma, and for CMV oral valgancyclovir. Inj. meropenem and moxifloxacin was prescribed to cover secondary bacterial infection including atypical microorganism empirically and she was recovered. To our knowledge this is first case of transplant patient suffered concomitant COVID and CMV infection.

\section{References}

1. World Health Organization. Coronavirus disease 2019 (COVID-19) Situation Report - 44. https://www.who.int/docs/default-source/ coronaviruse/situation-reports/20200304-sitrep-44-COVID-19. pdf? sfvrsn=783b4c9d_6

2. Elias M, Pievani D, C.COVID-19 Infection in Kidney Transplant Recipients: Disease Incidence and Clinical Outcomes

3. Banerjee D, Popoola J, Shah S, Chis Ster I, Quan V. COVID-19 infection in kidney transplant recipients. doi: https://doi.org/10.1016/j.kint.2020.03.018

4. Yang X, Yu Y, Xu J, et al. Clinical course and outcomes of critically ill patients with SARS-CoV-2 pneumonia in Wuhan, China: a single-centered, retrospective, observational study [e-pub ahead of print]. Lancet Respir Med.https://doi.org/10.1016/ S2213-2600(20) 30079-5. Accessed March 27, 2020.

5. Zhou F, Yu T, Du R, Fan G, Liu Y, Liu Z, et al. Clinical course and risk factors for mortality of adult inpatients with COVID-19 in Wuhan, China: a retrospective cohort study. Lancet. 2020; 395:1054-62. Erratum in: Lancet. 2020; 395:1038. Erratum in: Lancet. 2020; 3951038.

6. Global Outcomes (KDIGO) stage 1 five patients with KDIGO COVID-19 Infection in Kidney Transplant Recipients: Disease Incidence and Clinical Outcomes JASN 31: cec-ccc, 2020. doi: https://doi.org/ 10.1681/ ASN.2020050639

7. López V, Vázquez T, Alonso-Titos J, Cabello M, Alonso A, Beneyto I, et al. Recommendations on management of the SARS-CoV-2 coronavirus pandemic (COVID-19) in kidney transplant patients. Nefrologia. 2020: S0211-6995;30037-0.

8. Akalin E, Azzi Y, Bartash R, Seethamraju H, Parides M, Hemmige V, et al. COVID-19 and Kidney Transplantation. N Engl J Med. 2020. Epub ahead of print.

9. Naeem S, Gohh R, Bayliss G, Cosgrove C, Farmakiotis D, Merhi B, Morissey P, Osband A, Jeffrey A, Bailey, Sweeney J, Rogers R. Successful recovery from COVID-19 in three kidney transplant recipients who received convalescent plasma therapy. https://doi.org/10.1111/tid.13451

10. Maggione U, Abramowicz D, Crespo M, Christophe M, Mjoenn G et al How should I manage immunosuppression in a kidney transplant patient with COVID- 19? An ERA- EDTA DESCARTES expert opinion. Nephrol Dial transplant.2020jun;35(6):899-904. 